\title{
ChemComm
}

\section{Adsorbed and near-surface structure of ionic liquids determines nanoscale friction $\dagger$}

Cite this: Chem. Commun., 2013,
49, 6797

Received 17th April 2013

Accepted 11th June 2013

DOI: $10.1039 / c 3 c c 42844 c$

\author{
Aaron Elbourne, $\ddagger^{\mathrm{a}}$ James Sweeney, $t^{\mathrm{a}}$ Grant B. Webber, ${ }^{a}$ Erica J. Wanless, ${ }^{a}$ \\ Gregory G. Warr, ${ }^{\text {b }}$ Mark W. Rutland ${ }^{\mathrm{cd}}$ and Rob Atkin ${ }^{\star a}$
}

www.rsc.org/chemcomm

\begin{abstract}
Surface-adsorbed and near-surface ion layer structure controls nanotribology in the silica-propylammonium nitrate (PAN)-mica system. Atomic Force Microscopy (AFM) imaging and normal force curves reveal that the normal load dictates the number of interfacial ion layers and the lateral layer structure. Shear force measurements show the lubricity of the interface changes with the number, and lateral structure, of the confined ion layer(s).
\end{abstract}

Ionic Liquids (ILs) are salts with melting points less than $100{ }^{\circ} \mathrm{C} .{ }^{1}$ They have recently attracted a large amount of attention due to their physical properties including negligible vapour pressures, ${ }^{2}$ high thermal conductivities ${ }^{3}$ and heat capacities, ${ }^{4}$ high temperature stability, ${ }^{5}$ and wide electrochemical windows. ${ }^{5}$ ILs are designer solvents because their miscibility and solvency can be matched to practical applications by changing functional groups in the cation or anion. ${ }^{1,6-8}$ The first IL reported, ethylammoniun nitrate (EAN), is classified as a protic IL because it is synthesised by proton transfer from an acid to a base. ${ }^{9}$ PAN, ${ }^{10}$ which is the focus of this study, has an alkyl chain one $\mathrm{CH}_{2}$ unit longer. The effect of the longer alkyl chain on IL nanostructure in the bulk ${ }^{11}$ and normal to the interface $^{12}$ has been studied, but its effect on the lateral surface morphology, and PAN's ability to lubricate sliding surfaces, has not previously been probed.

Many ILs are nanostructured in the bulk and at interfaces. This nanostructure results from the forces between ions, which include electrostatic, van der Waals, hydrogen bonding and solvophobic components. ${ }^{13-15}$ Solvophobicity in ILs is similar

\footnotetext{
${ }^{a}$ Centre for Advanced Particle Processing and Transport, The University of Newcastle, Callaghan, NSW 2308, Australia. E-mail: Rob.Atkin@newcastle.edu.au; Fax: +61 2 49215472; Tel: +61249217107

${ }^{b}$ School of Chemistry, The University of Sydney, NSW 2006, Australia. E-mail: Gregory.Warr@sydney.edu.au; Fax: +61 2 93513329; Tel: +61 293512106 ${ }^{c}$ Surface and Corrosion Science, School of Chemical Science and Engineering, KTH - Royal Institute of Technology, Stockholm, Sweden

${ }^{d}$ YKI, Stockholm, Sweden. E-mail: Mark@kth.se; Tel: +4687909914

$\dagger$ Electronic supplementary information (ESI) available: Details of IL synthesis, AFM experimental parameters and supplementary data. See DOI: 10.1039/ c3cc42844c

$\ddagger$ These authors contributed equally to this manuscript.
}

to hydrophobicity in water, and causes alkyl chains to cluster together, segregated away from charged groups, resulting in polar and apolar domains in the IL. Small angle neutron diffraction experiments have shown that segregation of polar and apolar domains in PAN yields a bicontinuous $L_{3}$-sponge phase-like bulk liquid structure. ${ }^{11}$

IL interfacial nanostructure perpendicular to a solid surface has been studied extensively ${ }^{12,16-18}$ using AFM normal force curves. Steps in these force curves correspond to displacement of ion layers from between the surface and the sharp AFM tip or silica colloid probe. The force required to displace layers increases with decreasing separation, indicating the increasing strength of IL interfacial structure closer to the surface. ${ }^{12,19}$ IL interfacial structure may be considered to consist of three regions: the innermost layer, which consists of ions in contact with the solid surface; the near-surface transition zone, through which the interfacial structure decays to that of the bulk; and the bulk liquid..$^{20,21}$

ILs have attracted considerable research interest as lubricants over the last decade. ${ }^{22-25}$ Similar to high-performance lubricants such as perfluoropolyethers (PFPEs), ILs have low vapour pressures and high thermal stabilities. However, ILs are electrically conductive, meaning that they can be used to lubricate electrical contacts; PFPEs cannot because they are insulators. ${ }^{26}$

Here soft contact AFM imaging using sharp tips has been used to probe surface and near-surface IL structure at the muscovite mica-PAN interface, and the corresponding nanotribological (shear force) response determined using colloid probe AFM. A sharp AFM tip allows high resolution images of the IL lateral structure to be obtained, while the well-defined spherical geometry of the colloid probe enables accurate determination of the corresponding shear forces. Combining the findings from these two techniques allows the variations in shear force with normal load to be correlated with the interfacial IL structure. These results are compared to those obtained for $\mathrm{EAN}^{24,27}$ to reveal the impact of ion structure on near-surface lateral morphology and thus lubricity.

Fig. 1 (left, top) shows the apparent separation between a $5 \mu \mathrm{m}$ diameter silica colloid probe and a mica surface immersed in PAN as the normal load is increased. The normal force increases 

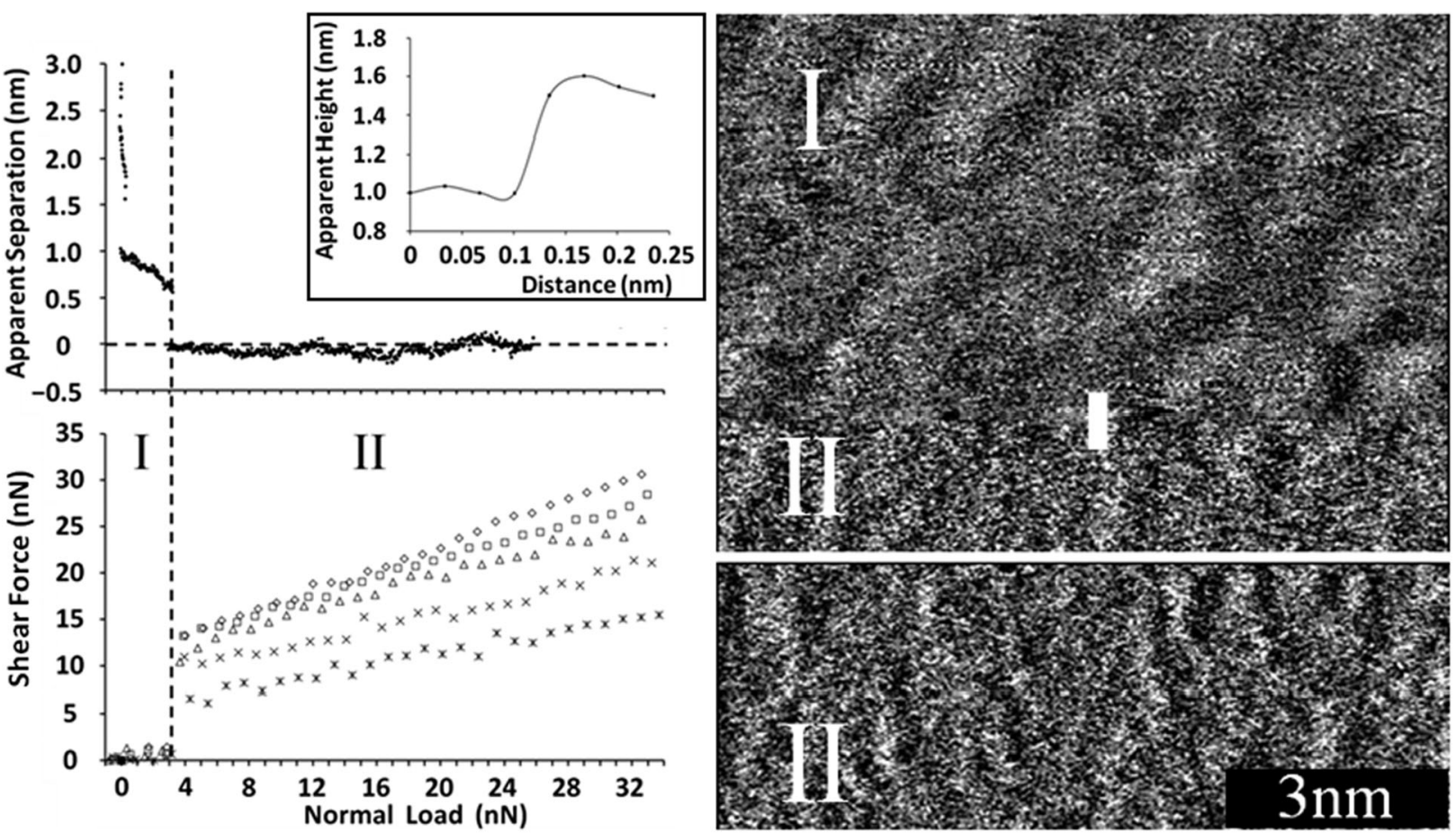

Fig. 1 (left) (top) Apparent separation between a mica surface and the silica colloid probe as a function of normal load for the mica-PAN system. (bottom) Shear force as a function of normal load for various sliding velocities using a colloid probe. Stars: $5 \mu \mathrm{m} \mathrm{s}^{-1}$, crosses: $10 \mu \mathrm{m} \mathrm{s}^{-1}$, triangles: $20 \mu \mathrm{m} \mathrm{s}{ }^{-1}$, squares: $30 \mu \mathrm{m} \mathrm{s}{ }^{-1}$, diamonds: $40 \mathrm{um} \mathrm{s}^{-1}$. The dashed vertical line delineates regions I and II (see text). (right) Sharp AFM tip images of the PAN-mica interface. The black $3 \mathrm{~nm}$ scale is valid for both images. In (I) the sharp tip is imaging the final transition zone layer in "soft" contact mode. While the image was being taken the imaging force increased, rupturing the layer and in (II) the sharp tip images the innermost layer at zero apparent separation. A section analysis for the sharp AFM tip moving from I to II is shown as an inset to the force curve (top left).

smoothly at large separations before exhibiting two discrete jumps and two subsequent layers. These are primarily a consequence of the mica surface and not associated with the silica colloid probe, because the silica surface is both rougher and has a lower surface charge density. ${ }^{12,24}$ Note that zero separation in an AFM force curve is determined from the compliance region in the raw force data and does not necessarily correspond to contact between the surface of the colloid probe and the mica substrate in IL systems. ${ }^{12,17}$ The force curves are similar to those obtained previously using a sharp AFM tip. ${ }^{12}$ Whereas analogous force measurements for EAN on mica revealed 4 or 5 steps $0.5 \mathrm{~nm}$ apart, attributed to expulsion of ion pair layers from between the sharp AFM tip and the surface, ${ }^{24}$ the near-surface PAN layers are more compressible due to the greater molecular flexibility of the propyl chains of the cation. ${ }^{12}$

Sharp AFM tips were used to obtain soft contact ${ }^{28}$ images of the PAN-mica system at various normal loads, $c f$. Fig. 1 (right). The upper section of the top image shows the near-surface transition zone layer (I), which has a distinct, undulating structure with a periodicity of about $1 \mathrm{~nm}$, and is evocative of PAN's bulk nanostructure. ${ }^{11}$ The features in this image are more pronounced than those reported previously for the same layer in EAN. ${ }^{27}$ This is a consequence of the longer alkyl chain of PAN producing a stronger solvophobic interaction and more pronounced bulk liquid structure. ${ }^{11,29}$

About three quarters of the way down the image (Fig. 1, top, right) instrumental drift increases the imaging force such that the compressible transition zone layer is ruptured and the sharp AFM tip jumps inward to the innermost layer (II). The height profile (inset to Fig. 1, left, top), taken along the vertical white line where the image appearance changes, shows that the height difference between the last transition zone and innermost layer is $\sim 0.5 \mathrm{~nm}$, consistent with both the step size in the normal force curve and the PAN cation-anion pair diameter $\left(\sim 0.56 \mathrm{~nm}^{12}\right)$. At these higher imaging forces worm-like lateral structures running perpendicular to the fast scan direction in the innermost layer are clearly visible in Fig. 1 (full image is shown in ESI, $\uparrow$ Fig. C). These structures scaled and rotated correctly with scan size and scan angle respectively, confirming they are not image artefacts. Their periodicity, calculated from the $2 \mathrm{D}$ Fourier transform, was $0.49 \mathrm{~nm}$. This is the same as the mica lattice spacing $\left(0.52 \mathrm{~nm}^{30}\right)$ within experimental error, and clearly much smaller than the transition zone layer structure.

This worm-like morphology arises from a balance between the electrostatic attraction of the cation's ammonium group for the fixed mica charged sites, and solvophobic attractions between exposed cation chains. A freshly cleaved mica surface consists of negatively charged surface groups in a hexagonal lattice, approximately half of which are neutralised by potassium ions. When PAN and mica come into contact, the $\mathrm{PA}^{+}$adsorbs to available surface charged sites, and competes with potassium ions for the occupied charged sites. Because the $\mathrm{PA}^{+}$concentration in the AFM cell is extremely high, and $\mathrm{PA}^{+}$is surface active, ${ }^{11,12}$ it is reasonable to assume that potassium is effectively expelled from all surface sites. Because the mica surface area is small, the resultant bulk potassium concentration in the cell is very low. 
The space between charged sites on the mica surface is sufficient to allow additional cations to associate solvophobically, but to maintain charge neutrality an additional anion is also adsorbed into the innermost layer. The presence of these anions places an additional constraint on the self-assembly process, inhibiting alkyl chain association in certain directions, resulting in the worm-like structures. As such, the worm structures are the self-assembled morphology that maximises attractions between cation alkyl chains and satisfies the electrostatic requirements of both the surface and the ions. ${ }^{27}$

The shear force as a function of normal load plot (Fig. 1, left, bottom) shows two distinct regions, which correspond to the colloid probe sliding along either a transition zone layer (I) or the innermost layer (II). In region I, when the normal force is low and transition zone layer(s) are present between the colloid probe and the surface, the resistance to sliding is very low. At the beginning of region II ( $\sim 3 \mathrm{nN}$ normal load), a step change occurs in the separation, with the shear force jumping abruptly from $\sim 1 \mathrm{nN}$ to between $7 \mathrm{nN}$ and $13 \mathrm{nN}$, depending on sliding velocity. This change occurs as the colloid probe moves from being in contact with the sponge-like near-surface layer to the worm-like innermost layer, which is much less lubricating.

In region II, the shear force increases with normal load. The friction coefficient increases logarithmically with sliding speed; $5 \mu \mathrm{m} \mathrm{s}^{-1}: 0.33,10 \mu \mathrm{m} \mathrm{s}^{-1}: 0.39,20 \mu \mathrm{m} \mathrm{s}^{-1}: 0.50,30 \mu \mathrm{m} \mathrm{s}^{-1}$ : 0.53 , and $40 \mu \mathrm{m} \mathrm{s}^{-1}: 0.63$. Plots of shear force (Fig. D, ESI + ) and friction coefficient (Fig. E, ESI $\dagger$ ) versus sliding velocity demonstrating the logarithmic dependence are provided in the ESI. $†$ Corresponding data was obtained for EAN when the colloid probe was sliding against the worm-like structures, ${ }^{24}$ and has also been observed in molecular lubricants ${ }^{31}$ and polymers, ${ }^{32}$ and is attributed to an activated, discontinuous sliding process. $^{24}$

In the EAN-mica system ${ }^{24}$ a second transition occurs at high normal loads $(>\sim 13 \mathrm{nN})$. There is a small compressive step, above which the friction coefficient decreases and imaging confirms that the worm-like structure of the innermost layer is replaced by a smoother layer of cations strongly adsorbed to mica lattice charged sites. ${ }^{27}$ In PAN however the worm-like structures remain in place up to at least $35 \mathrm{nN}$ normal load as the stronger solvophobic attractions between propyl groups prevent the expulsion of the solvophobically adsorbed cations from the innermost layer.

The unique combination of soft contact images and shear force measurements used in this work enables emphatic conclusions to be drawn regarding the effect of normal force and lateral structure on lubricity. For PAN, two different surface structures can be imaged (sponge-like and worm-like) and there are two regions in the shear force data. For EAN three different structures are noted (sponge-like, worm-like, and compressed electrostatically bound ions), ${ }^{27}$ and there is a third region in the shear force data ${ }^{24}$ at high force. The shear response for PAN and EAN in regions I and II is similar, confirming that the probe is sliding against transition zone sponge layers in region I, and the worm-like surface layer structure in region II. Region III in the shear force data for EAN begins when the normal force is increased to values high enough to cause (relatively weakly) solvophobically adsorbed cations to be expelled from the innermost layer, i.e., as the surface structure changes from worm-like to electrostatically bound cations only.

This means that the cation alkyl chain length can be used to control the strength of solvophobic attraction, and therefore the lateral morphology and lubricity; ILs allow intimate control over lubricity because changes in normal load change the lateral surface morphology.

\section{Notes and references}

1 R. Hayes, G. G. Warr and R. Atkin, Phys. Chem. Chem. Phys., 2010, 12, 1709-1723.

2 T. L. Greaves, A. Weerawardena, C. Fong and C. J. Drummond, J. Phys. Chem. B, 2007, 111, 4082-4088.

3 M. E. Van Valkenburg, R. L. Vaughn, M. Williams and J. S. Wilkes, in Fifteenth Symposium on Thermophysical Properties, Boulder, Colorado, USA, 2003.

4 P.-Y. Lin, A. N. Soriano, R. B. Leron and M.-H. Li, J. Chem. Thermodyn., 2010, 42, 994-998.

5 F. Endres and S. Z. El Abedin, Phys. Chem. Chem. Phys., 2006, 8, 2101-2116.

6 T. L. Greaves and C. J. Drummond, Chem. Rev., 2008, 108, 206-237.

7 R. Atkin, S. M. C. Bobillier and G. G. Warr, J. Phys. Chem. B, 2010, 114, 1350-1360.

8 R. Atkin, L. M. De Fina, U. Kiederling and G. G. Warr, J. Phys. Chem. $B, 2009,113,12201-12213$.

9 P. Walden, Bull. Acad. Imp. Sci. St.-Petersbourg, 1914, 1800.

10 C. F. Poole, B. R. Kersten, S. S. J. Ho, M. E. Coddens and K. G. Furton, J. Chromatogr., 1986, 352, 407-425.

11 R. Hayes, S. Imberti, G. G. Warr and R. Atkin, Phys. Chem. Chem. Phys., 2011, 13, 13544-13551.

12 R. Atkin and G. G. Warr, J. Phys. Chem. C, 2007, 111, 5162-5168.

13 R. Hayes, S. Imberti, G. G. Warr and R. Atkin, Angew. Chem., Int. Ed., 2013, 52, 4623-4627.

14 H. Tokuda, K. Hayamizu, K. Ishii, M. A. B. H. Susan and M. Watanabe, J. Phys. Chem. B, 2004, 108, 16593-16600.

15 A. Ray, Nature, 1971, 231, 313-315.

16 R. Hayes, S. Z. El Abedin and R. Atkin, J. Phys. Chem. B, 2009, 113, 7049-7052.

17 D. Wakeham, R. Hayes, G. G. Warr and R. Atkin, J. Phys. Chem. B, 2009, 113, 5961-5966.

18 J. A. Smith, O. Werzer, G. B. Webber, G. G. Warr and R. Atkin, J. Phys. Chem. Lett., 2010, 1, 64-68.

19 S. Perkin, T. Albrecht and J. Klein, Phys. Chem. Chem. Phys., 2010, 12, 1243-1247.

20 R. Atkin, N. Borisenko, M. Druschler, S. Z. El Abedin, F. Endres, R. Hayes, B. Huber and B. Roling, Phys. Chem. Chem. Phys., 2011, 13, 6849-6857.

21 R. Hayes, D. Wakeham and R. Atkin, in Ionic Liquids Uncoiled: Critical Expert Overviews, ed. K. R. Seddon, N. V. Plechkova and R. D. Rogers, John Wiley \& Sons, 2012, p. 438.

22 M. Palacio and B. Bhushan, Tribol. Lett., 2010, 40, 247-268.

23 S. Perkin, T. Albrecht and J. Klein, Phys. Chem. Chem. Phys., 2010, 12, 1243-1247.

24 O. Werzer, E. D. Cranston, G. G. Warr, R. Atkin and M. W. Rutland, Phys. Chem. Chem. Phys., 2012, 14, 5147-5152.

25 J. Sweeney, F. Hausen, R. Hayes, G. B. Webber, F. Endres, M. W. Rutland, R. Bennewitz and R. Atkin, Phys. Rev. Lett., 2012, 109, 155502.

26 B. Bhushan, M. Palacio and B. Kinzig, J. Colloid Interface Sci., 2008, 317, 275-287.

27 J. J. Segura, A. Elbourne, E. J. Wanless, G. G. Warr, K. Voitchovsky and R. Atkin, Phys. Chem. Chem. Phys., 2013, 15, 3320-3328.

28 H. N. Patrick, G. G. Warr, S. Manne and I. A. Aksay, Langmuir, 1997, 13, 4349-4356.

29 R. Atkin and G. G. Warr, J. Phys. Chem. B, 2008, 112, 4164-4166.

30 B. James and H. James, in Dekker Encyclopedia of Nanoscience and Nanotechnology, Second Edition - Six Volume Set (Print Version), CRC Press, 2008, pp. 2211-2228.

31 M. He, A. S. Blum, G. Overney and R. M. Overney, Phys. Rev. Lett., 2002, 88, 154302.

32 T. Bouhacina, J. P. Aime, S. Gauthier, D. Michel and V. Heroguez, Phys. Rev. B: Condens. Matter Mater. Phys., 1997, 56, 7694-7703. 